\title{
Introduction: Reconsidering Disorders of Consciousness in Light of Neuroscientific Evidence
}

\author{
Ralf J. Jox • Katja Kuehlmeyer
}

Received: 22 August 2011 / Accepted: 7 September 2011 /Published online: 23 September 2011

(C) The Author(s) 2011. This article is published with open access at Springerlink.com

\begin{abstract}
Disorders of consciousness pose a substantial ethical challenge to clinical decision making, especially regarding the use of life-sustaining medical treatment. For these decisions it is paramount to know whether the patient is aware or not. Recent brain research has been striving to assess awareness by using mainly functional magnetic resonance imaging. We review the neuroscientific evidence and summarize the potential and problems of the different approaches to prove awareness. Finally, we formulate the crucial ethical questions and outline the different articles in this special issue on disorders of consciousness.
\end{abstract}

Keywords Disorders of consciousness - Vegetative state $\cdot$ Minimally conscious state $\cdot$ End-of-life decisions $\cdot$ Functional magnetic resonance imaging

Progress in medicine allows more and more people in critical conditions to be kept alive. Some of these patients, however, do not fully regain consciousness due to substantial brain damage. If life-sustaining treatment is applied, they may remain in chronic

\footnotetext{
R. J. Jox $(\bowtie) \cdot$ K. Kuehlmeyer

Institute for Ethics, History and Theory of Medicine, University of Munich,

Lessingstraße 2,

D-80336 München, Germany

e-mail: ralf.jox@med.lmu.de

URL: www.egt.med.uni-muenchen.de
}

disorders of consciousness for decades, living in a vegetative state (VS) or a minimally conscious state (MCS). The VS is characterized by preserved sleepwake-cycles and spontaneous breathing, which is due to a largely intact brainstem function, yet there are no signs of behavioural reactions to stimuli, interaction, or communication. It is assumed that a patient in the VS is awake but not aware of himself or his surroundings [1]. Due to the pejorative connotation of the term 'vegetative' the European Task Force on Disorders of Consciousness has recently suggested the term unresponsive wakefulness syndrome (UWS) [2]. In contrast to the VS, a patient in MCS displays inconsistent, but reproducible purposeful behaviour, heralded by subtle signs such as visual pursuit, correct object use, intelligible verbalisation, or following simple commands [3]. It is assumed that awareness is preserved in the MCS, at least at a basic level. Both disorders of consciousness have to be strictly distinguished from the locked-in state (LiS). Patients in a LiS are usually fully conscious, but cannot express themselves due to extensive paralysis, yet many are able to use vertical eye or eyelid movements to communicate.

The LiS demonstrates that awareness does not necessarily hinge on the ability to express oneself or interact with others, be it verbally or through behaviour. Therefore, the absence of evidence for awareness is not tantamount to evidence for the absence of awareness. How can we then determine whether a person is aware or not? Is it possible to 
prove the absence or presence of awareness in patients diagnosed with VS or MCS using neuroscientific technology? Many researchers have tried to answer this question by presenting stimuli to patients and registering the reaction using either electrophysiological or functional brain imaging techniques. They not only used intensive stimuli such as pain, but also personally meaningful stimuli such as the verbal presentation of the patient's own name. Some patients showed reactions that were similar to healthy controls [4, 5]. Selected patients have also been shown to activate the neural correlates of speech comprehension in functional magnetic resonance imaging (fMRI) [6]. Yet, these passive paradigms only demonstrate that a certain brain response follows a given stimulus. Whether this response is accompanied by awareness, however, is beyond the reach of these experiments. It is well conceivable that cortical networks for pain processing or auditory processing may function independently of awareness. After all, we know from our daily life that we are able to unconsciously perform complex actions like brushing our teeth or driving a car. Salient stimuli, like being presented one's own name, may prompt automatic brain responses due to facilitated synaptic projections.

Thus, brain researchers moved on to active paradigms. Here, assuming that they are aware and understand verbal language, patients are asked to perform mental tasks (e.g. counting, imagining). Patients in VS and MCS were instructed to imagine playing tennis or walking through their homes while lying in the fMRI scanner [7]. These tasks produce two distinct and reliable fMRI patterns in healthy subjects. In a recently published case series, the fMRI demonstrated the same two activity patterns in five VS patients in their response to the tasks [8]. The authors took this as proof that the patients were able to wilfully modulate their brain activity, alternating between the tennis and the navigation tasks. The rather bold conclusion, which stated that these patients consciously and voluntarily chose to activate a certain brain network, was heavily criticised $[9,10]$.

The next step was to try to establish a basic form of communication with patients. They were asked to use the two mental activities (playing tennis and navigation) to signal "yes" or "no" to presented questions [8]. Indeed, one VS patient was reported to have responded with the correct brain activity pattern when asked biographical questions, for example whether the name of his father was Alexander or Thomas. This form of communication, however, has only been demonstrated by one of the 54 patients in the study [8]. Obviously, it is a rather small subset of VS patients that shows this remarkable ability to communicate. Moreover, this approach must promise several functions in order to prove the presence of consciousness: it requires, for example, an intact auditory pathway, functioning language networks of the cortex, and uninjured cortical areas for movement planning and navigation. Given the fact that all of these cognitive capacities need extensive areas of the brain to be intact, it is highly improbable that these capacities will be preserved in many brain injured patients. This means, however, that the approach will yield false negative results regarding the question whether the patient is aware or not. This might explain that many MCS patients in the study were not able to follow the task, even though they were presumed to be aware according to behavioural testing [8]. This also raises the question on what basis patients should be diagnosed: on the traditional basis of behavioural tests like the Coma Recovery Scale-Revised, or on the basis of functional imaging studies using active paradigms?

This is why a new approach to prove awareness is a purely objective one that does not presuppose any cognitive capacities: if people undergo an fMRI scan without a given task, just "lying and thinking of nothing," they exhibit activity in two so-called resting state networks that alternate every few seconds. Researchers hypothesize that these networks might signify the awareness of oneself and of one's surroundings [11]. It has already been discovered that the activation of those networks are reduced in patients with disorders of consciousness [12]. The problem with this approach is, on the other hand, that the underlying hypothesis that these networks necessarily herald awareness is still unproven. This touches on the epistemological problem that a subjective entity such as awareness cannot be proven purely by objective measurement - without the person intersubjectively revealing himself as a conscious being.

Although many of these neuroscientific results have not been rigorously confirmed, it has already been discussed what they mean for the ethical evaluation of the patient's condition and how they should influence the clinical decision concerning whether to keep patients alive or not [13]. It has even 
been speculated whether the communicative fMRI paradigm could be used, clinically, to question patients about their wishes for life-sustaining treatment [14]. Moreover, how does the proof of awareness itself change the decision concerning lifesustaining treatment? Critics argue that rudimentary consciousness does not directly lead to a higher quality of life or alter the patient's moral status [13]. Indeed, as conscious patients can also feel pain and experience suffering, there may be even more grounds for withdrawing life support [15]. This danger would have to be weighed against the chance of later rehabilitation and the possibility of patients having new and positive experiences. Yet, these neuroscientific results do provide the hope of being able to communicate with brain injured patients in the future by means of so-called brain-computer interfaces [16].

Some of these ethical and philosophical questions that have evolved through the exciting new developments in neuroscience are addressed in this special issue of Neuroethics. The articles are derived from an international neuroethics workshop for young researchers from different disciplines that was funded by the German Ministry of Education and Research and held in March 2011 in Munich, Germany. Kirsten Brukamp explores what these new research findings mean for the diagnosis of brain-injured patients. She asserts that the communicative ability of all patients with disorders of consciousness should be tested using fMRI. Orsolya Friedrich discusses how the neuroscientific data might influence end-of-life decision making from a philosophical point of view. Catherine Rodrigue et al. present the results of a qualitative interview study with Canadian health care professionals on clinical diagnosis, prognosis, and end-of-life decision making. Athena Demertzi et al. report the findings of their survey of a large sample of healthcare professionals from 32 European countries and suggest that opinions on pain perception mediate attitudes towards end-of-life decisions in VS patients. Finally, Christopher Mahar argues, from a Catholic point of view, why society should be obliged to care for patients in the VS. The authors portray how the new findings in brain research may influence and even improve clinical practice. On the other hand, they also point to the sobering limits in transferring the marvelous neuroscience to the patient's bedside and urge the need for a broad societal discussion about the optimal care for these highly vulnerable patients.
Acknowledgement We gratefully acknowledge grant support by the German Ministry of Education and Research (FKZ 01GP0801) and thank Dorothee Wagner von Hoff for proofreading.

Open Access This article is distributed under the terms of the Creative Commons Attribution Noncommercial License which permits any noncommercial use, distribution, and reproduction in any medium, provided the original author(s) and source are credited.

\section{References}

1. Bernat, J.L. 2009. Chronic consciousness disorders. Annu Rev Med 60: 381-392.

2. Laureys, S., G.G. Celesia, F. Cohadon, et al. 2010. Unresponsive wakefulness syndrome: a new name for the vegetative state or apallic syndrome. $B M C \mathrm{Med}$ 8: 68 .

3. Giacino, J.T. 2005. The minimally conscious state: defining the borders of consciousness. Prog Brain Res 150: 381-395.

4. Boly, M., M.E. Faymonville, C. Schnakers, et al. 2008. Perception of pain in the minimally conscious state with PET activation: an observational study. Lancet Neurol 7: 1013-1020.

5. Perrin, F., C. Schnakers, M. Schabus, et al. 2006. Brain response to one's own name in vegetative state, minimally conscious state, and locked-in syndrome. Arch Neurol 63: $562-569$.

6. Coleman, M.R., M.H. Davis, J.M. Rodd, et al. 2009. Towards the routine use of brain imaging to aid the clinical diagnosis of disorders of consciousness. Brain 132: 2541-2552.

7. Owen, A.M., M.R. Coleman, M. Boly, et al. 2006. Detecting awareness in the vegetative state. Science 313: 1402.

8. Monti, M.M., A. Vanhaudenhuyse, M.R. Coleman, et al. 2010. Willful modulation of brain activity in disorders of consciousness. $N$ Engl J Med 362: 579-589.

9. Greenberg, D.L. 2007. Comment on "Detecting awareness in the vegetative state". Science 315: 1221. author reply 1221.

10. Nachev, P., and M. Husain. 2007. Comment on "Detecting awareness in the vegetative state". Science 315: 1221. author reply 1221.

11. Vanhaudenhuyse, A., A. Demertzi, M. Schabus, et al. 2011. Two distinct neuronal networks mediate the awareness of environment and of self. J Cogn Neurosci 23: 570-578.

12. Vanhaudenhuyse, A., Q. Noirhomme, L.J. Tshibanda, et al. 2010. Default network connectivity reflects the level of consciousness in non-communicative brain-damaged patients. Brain 133: 161-171.

13. Levy, N., and J. Savulescu. 2009. Moral significance of phenomenal consciousness. Prog Brain Res 177: 361-370.

14. Ropper, A.H. 2010. Cogito ergo sum by MRI. $N$ Engl $J$ Med 362: 648-649.

15. Wilkinson, D.J., G. Kahane, M. Horne, et al. 2009. Functional neuroimaging and withdrawal of life-sustaining treatment from vegetative patients. $J$ Med Ethics 35: 508-511.

16. Kubler, A., and N. Neumann. 2005. Brain-computer interfaces-the key for the conscious brain locked into a paralyzed body. Prog Brain Res 150: 513-525. 